Check for updates

Cite this: New J. Chem., 2020, 44, 19144

Received 19th August 2020

Accepted 20th October 2020

DOI: 10.1039/d0nj04187d

rsc.li/njc

\section{Boronic acids for functionalisation of commercial multi-layer graphitic material as an alternative to diazonium salts $\dagger$}

\author{
Rachel L. McLaren, (D) ${ }^{a}$ Christian J. Laycock, (D) a David J. Morgan (D) bc and \\ Gareth R. Owen (iD)*a
}

\begin{abstract}
A novel radical-based functionalisation strategy for the synthesis of functionalised commercially obtained plasma-synthesised multi-layer graphitic material (MLG) is presented herein. 4-(trifluoromethyl)phenyl boronic acid was utilised as a source of 4-(trifluoromethyl)phenyl radicals to covalently graft upon the graphitic surface of MLG. Such a methodology provides a convenient and safer route towards aryl radical generation, serving as a potential alternative to hazardous diazonium salt precusors. The structure and morphology of the functionalised MLG ( $\left.{ }^{\mathrm{Ar}} f-M L G\right)$ has been characterised using XPS, Raman, TGA, XRD, SEM, TEM and BET techniques. The XPS quantitative data and Raman spectra provide evidence of successful covalent attachment of 4-(trifluoromethyl)phenyl groups to MLG.
\end{abstract}

\section{Introduction}

Graphene describes an allotropic form of carbon consisting of a single layer hexagonal array of $\mathrm{sp}^{2}$ hybridised carbon atoms. The combination of stacked graphene planes makes up the well-known structure of graphite. Whilst graphite has been known for several hundred years, graphene itself was not isolated until 2004. ${ }^{1}$ Graphene possesses excellent mechanical, electrical, optical, thermal and biocompatible properties. A whole host of potential applications for these materials has been touted by both academia and industry. ${ }^{2,3}$ Although research on this material is still in its early stages of development, plenty of applications have been proposed which harness and exploit the aforementioned properties.

Notwithstanding all of the aforementioned properties of these materials, there are some significant challenges which hinder the practical application of graphene materials on any large or industrial scale. ${ }^{4}$ Graphene is a challenging material to work with due to its limited dispersibility within most solvents. Additionally, characterisation challenges are very common. ${ }^{5}$ Pristine graphene is only available in small laboratory scale

\footnotetext{
${ }^{a}$ School of Applied Science, University of South Wales, Treforest, CF37 4AT, UK. E-mail: gareth.owen@southwales.ac.uk

${ }^{b}$ Cardiff Catalysis Institute, School of Chemistry, Cardiff University, Cardiff, CF10 3AT, UK

${ }^{c}$ HarwellXPS, EPSRC National Research Facility for Photoelectron Spectroscopy, RCaH, Didcot, Oxon, OX11 OFA, UK

$\dagger$ Electronic supplementary information (ESI) available: Containing further spectroscopic and analytical details concerning the characterisation of MLG and ${ }^{\mathrm{Ar}}$-MLG. See DOI: 10.1039/d0nj04187d
}

quantities whilst larger scale industrially derived graphene contains many defects, oxygen functionalisation and are typically obtained as multi-layer graphitic material (MLG). ${ }^{6}$ Furthermore it is typically obtained with a broad distribution of sizes and morphologies.

Issues such as processability can be addressed via functionalisation. Functionalisation can also alter the properties of the material in a number of ways. For example, addition of new functional groups can enhance solubility and/or generate new composite materials with specifically designed properties. ${ }^{7,8}$ The incorporation of additional organic or inorganic moieties to the surface of such materials also creates steric repulsion between the sheets and stacks, thus providing an energy barrier against undesired clumping. ${ }^{9}$ Furthermore, covalent functionalisation can also alter the graphitic structure by introducing a band gap, thereby allowing the material to switch to lower conductance states. ${ }^{10}$ It is thus imperative to obtain new, safe routes to introduce covalent functionality to new materials. ${ }^{7,8}$

Over the 16 years since graphene was first isolated, a vast amount of research has been devoted to the development of methods towards covalently functionalised graphene. ${ }^{11-18}$ Many of these methodologies utilise hazardous conditions, such as diazonium salts. From a synthetic point of view, diazonium salts provide an excellent and straightforward source of radicals from which the only by-product is nitrogen gas. ${ }^{19}$ This offers a clean methodology for attaching functional groups to the material. Such radicals combine with radicals present on the surface to form new covalent bonds as demonstrated by a number of groups. ${ }^{20-27}$ Their application, however, does have some undesirable synthetic conditions which makes it challenging for large scale industrial 
production. Many diazonium salts decompose violently and can be sensitive to friction and shock. ${ }^{28}$ As a result, access to radicals through alternative precursors is desirable for the purpose of large-scale functionalisation of graphene and graphitic material.

Through an industrial collaboration, we embarked on an investigation to explore alternative starting materials which allow access to aryl radicals required for covalent functionalisation. From an industrial perspective, plasma-synthesised multilayer graphitic material ${ }^{29,30}$ is more accessible and as such there is significant value in finding synthetic methodologies for its derivatisation. As such we aimed to explore these more accessible materials for functionalisation as opposed to graphene. Herein, we wish to report a preliminary account of these investigations. We show that 4-(trifluoromethyl)phenyl radicals, accessed via oxidative conditions of 4-(trifluoromethyl)phenyl boronic acid, can be used to covalently functionalise the outer surfaces of plasma-synthesised MLG, thus forming 4-(trifluoromethyl)phenyl functionalised MLG ( ${ }^{\mathrm{Ar}} f$-MLG). We also explore the impact of carrying out this functionalisation by examining the changes in relation to the original material. The functionalised material was characterised using XPS, Raman, TGA, XRD, SEM, TEM, and BET spectroscopic and analytical methods.

\section{Results and discussion}

\section{Synthesis of ${ }^{\mathrm{Ar}} \boldsymbol{f}$-MLG}

As a means of exploring alternatives to generate aryl radicals in situ for the purposes of covalently functionalising MLG, we looked at aryl boronic acids as potential sources. Aryl boronic acids are well known for their applications in cross coupling reactions, leading to the formation of $\mathrm{C}-\mathrm{C}$ bonds. It has previously been demonstrated that aryl radicals can be generated from aryl boronic acids under various oxidative conditions, as an alternative strategy for forming $\mathrm{C}-\mathrm{C}$ bonds. For example, oxidants and combinations of oxidants such as $\mathrm{Mn}(\mathrm{OAc})_{3},{ }^{31} \mathrm{AgNO}_{3} /$ $\mathrm{K}_{2} \mathrm{~S}_{2} \mathrm{O}_{8},{ }^{32,33} \mathrm{Fe}(\mathrm{OTf})_{3} /\left({ }^{t} \mathrm{BuO}\right)_{2}{ }^{34}$ and $\left[\mathrm{Ni}(\mathrm{acac})_{2}\right] / \mathrm{K}_{3} \mathrm{PO}_{4} /\left({ }^{t} \mathrm{BuO}\right)_{2}{ }^{35}$ have all been employed for these purposes.

Baran recently reported the application of a mixture of silver nitrate and potassium persulfate for the formation of $\mathrm{C}-\mathrm{C}$ bonds. ${ }^{32,33}$ It has been postulated that the persulfate is reduced by the silver to form both $\left[\mathrm{SO}_{4}\right]^{2-}$ and $\left[\mathrm{SO}_{4}\right]^{\bullet-}$, which in turn react with the aryl boronic acid to generate aryl radicals (Scheme 1). ${ }^{32}$ The silver(II) is then reduced back to silver(I) as shown.

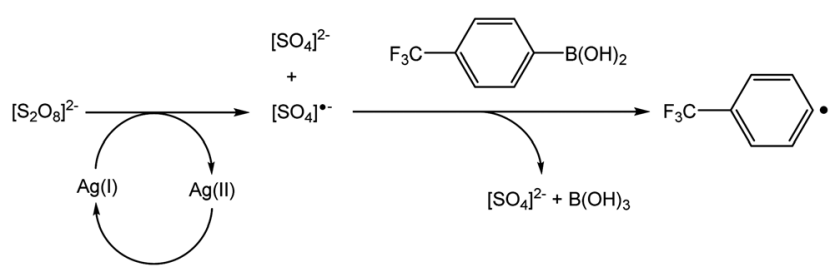

Scheme 1 Reported mechanism for the generation of aryl radicals using the approach by Baran and co-workers. ${ }^{32}$
The transformations proceed within a biphasic aqueous/DCM solvent system.

We utilised the Baran protocol for generating aryl radicals in the presence of MLG as a means of functionalising this material. For the purposes of aiding the subsequent characterisation, we chose 4-(trifluoromethyl)phenyl boronic acid as the aryl radical source. This precursor was chosen since it was reported to be most the efficient of those tested (due to the electron withdrawing nature of the $\mathrm{CF}_{3}$ group) ${ }^{32}$ and it contained fluorine which could be readily observed by X-ray photoelectron spectroscopy (XPS). Prior to the reaction with the graphene material, a control reaction was undertaken. In this control reaction, 4-(trifluoromethyl)phenyl boronic acid was reacted with silver nitrate and potassium persulfate in a 1:1 mixture of water and DCM (Scheme 2). We found that $44 \mathrm{~h}$ stirring at room temperature was sufficient for complete conversion of the starting material. Two products were separated from the DCM layer following work up. These products were identified as the homo-coupled product $4,4^{\prime}$-bis(trifluoromethyl)1,1'-biphenyl (A) and 4,4'-bis(trifluoromethyl)-1,1'-biphenyl ether (B) by NMR spectroscopy and mass spectrometry, in an approximate 1:1 ratio. The former product (A) was expected, whilst the oxygen-bridged compound (B) was not initially anticipated. We believe that this product originates from the radical species reacting with water to form phenol, which then undergoes a $\mathrm{C}-\mathrm{O}$ coupling reaction to form the ether product. It was important to confirm the identify of these products since they can also form intermolecular interactions with the surface of the material, which can confuse later interpretation and its characterisation.

With evidence of radical generation, we proceeded to repeat the same conditions in the presence of MLG with the aim of functionalising this material with the aryl groups (to form ${ }^{\mathrm{Ar}}$-MLG) with the expectation that both $4,4^{\prime}$-bis(trifluoromethyl)1,1'-biphenyl and 4,4'-bis(trifluoromethyl)-1,1'-biphenyl ether organic side products would also be present within the reaction mixture (Scheme 3). After $44 \mathrm{~h}$, the newly formed ${ }^{\mathrm{Ar}} f$-MLG was isolated from the reaction mixture. This involved purification steps to ensure that organic and inorganic by-products were mostly removed from aryl functionalised MLG ( ${ }^{\mathrm{Ar}} f$-MLG). The reaction mixture was centrifuged and the resulting powder was subjected to repeated dispersion/centrifugation cycles using large volumes of water, acetonitrile and DCM. The remaining

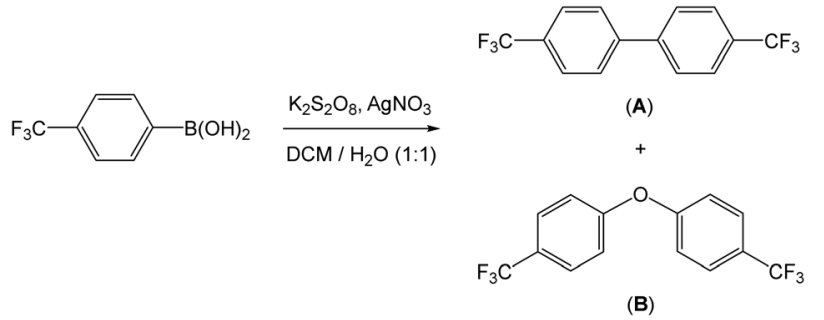

Scheme 2 Reaction of 4-(trifluoromethyl)phenyl boronic acid with silver nitrate and potassium persulfate in a biphasic water and DCM reaction. 


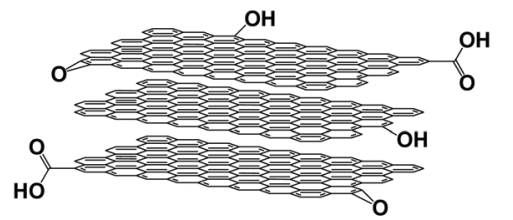

MLG

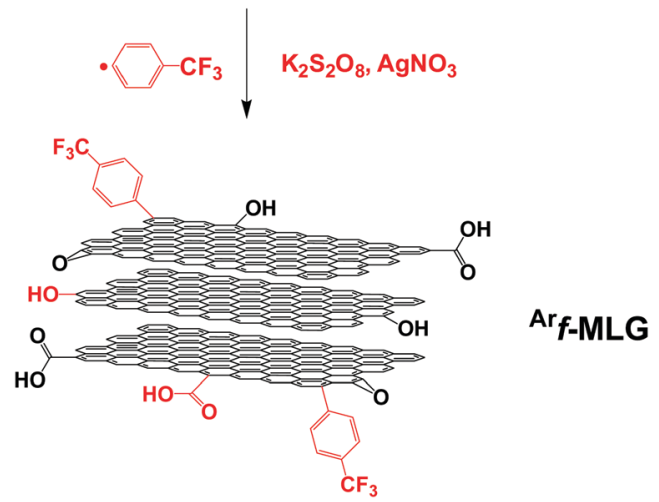

Scheme 3 Schematic representing the covalent attachment of 4-(trifluoromethyl)phenyl radicals to the MLG to form ${ }^{{ }^{A r}}$ - -MLG. The oxidising conditions leads to further oxidation of the material (see text for details). For clarity, MLG is drawn with only three layers.

solid material was then placed under high vacuum $\left(10^{-6}\right.$ bar $)$ for $168 \mathrm{~h}$.

\section{Analysis and characterisation of ${ }^{\mathrm{Ar}} \boldsymbol{f}$-MLG}

The new functionalised material, ${ }^{\mathrm{Ar}}$-MLG, was analysed and characterised via a range of techniques in comparison with the original MLG sample. This allowed us to examine the degree of functionalisation and its impact on the graphitic structure. The results acquired from these techniques are outlined below.

\section{X-ray photoelectron spectroscopy}

Both original and functionalised samples were examined by XPS, the results of which are presented in Fig. 1-4 and Table 1. Additional details and spectra are provided in the ESI, $\dagger$ in Fig. S1.

We initially characterised MLG and subsequently ${ }^{\mathrm{Ar}} f$-MLG to identify and compare surface characteristics, as well as the changes to its elemental composition. The data confirms changes to the elemental composition of the material upon functionalisation (Table 1 and Fig. 1). Most notable is the incorporation of fluorine into ${ }^{\mathrm{Ar}} f$-MLG with a relative atomic concentration of $3.5 \%$ (at $\%$ ) for the $\mathrm{F}$ 1s orbital.
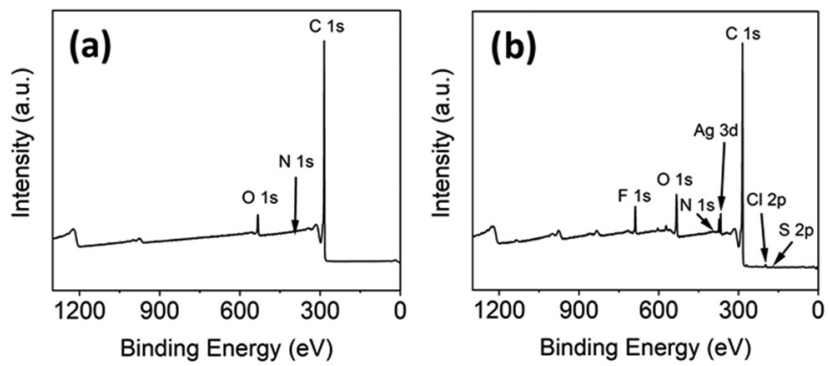

Fig. 1 XPS survey spectra of; (a) MLG and (b) ${ }^{A r} f-M L G$.
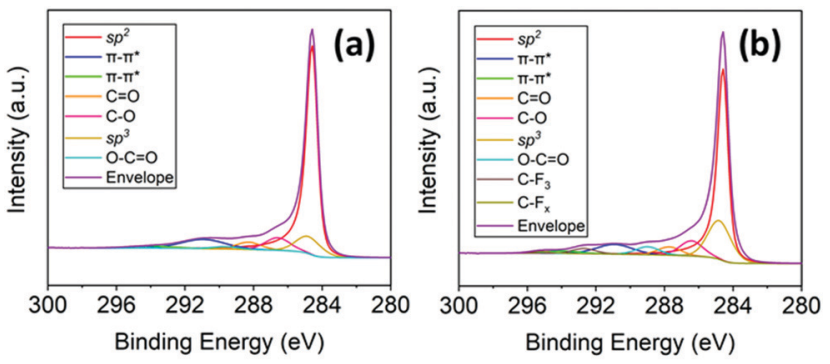

Fig. 2 Deconvoluted spectra of C 1 s orbital; (a) MLG and (b) ${ }^{A r} f-M L G$.
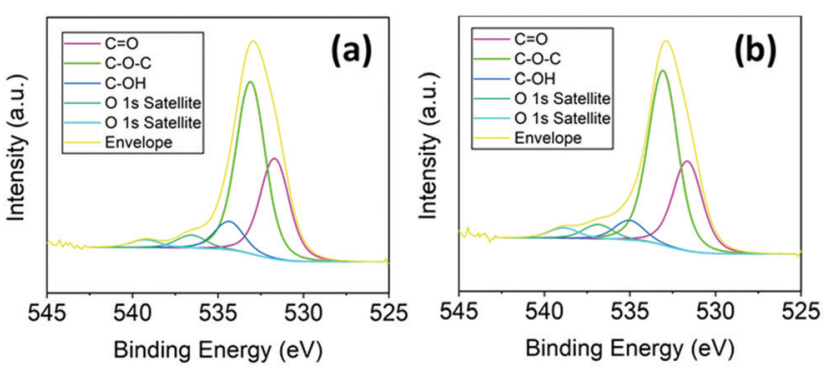

Fig. 3 Deconvoluted spectra of O 1s orbital; (a) MLG and (b) ${ }^{A r} f-M L G$.
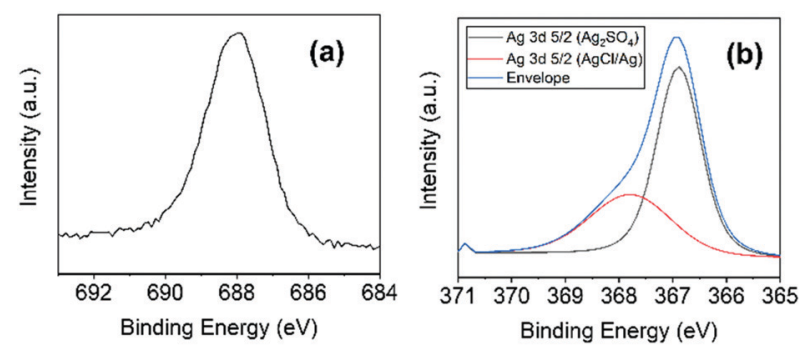

Fig. 4 XPS spectra of (a) F $1 \mathrm{~s}$ and (b) Ag $3 d_{5 / 2}$ orbital for ${ }^{A r} f-M L G$ at high resolution (deconvolution of the $3 \mathrm{~d}$ peak indicates presence of $\mathrm{Ag}, \mathrm{Ag}_{2} \mathrm{SO}_{4}$ and $\mathrm{AgCl}$.

Table 1 XPS surface elemental composition of $M L G,{ }^{A r} f-M L G$ and COx-MLG

\begin{tabular}{|c|c|c|c|}
\hline \multirow[b]{2}{*}{ Orbital } & \multicolumn{3}{|c|}{ Atomic concentration (\%) } \\
\hline & MLG & ${ }^{\mathrm{Ar}} f$-MLG & ${ }^{c} \mathrm{Ox}-\mathrm{MLG}$ \\
\hline $\mathrm{C} \mathrm{sp}^{2}$ & 64.3 & 47.5 & 51.2 \\
\hline$\pi-\pi^{*}$ & 10.3 & 7.5 & 8.7 \\
\hline $\mathrm{C}=\mathrm{O}$ & 3.8 & 3.4 & 4.3 \\
\hline $\mathrm{C}-\mathrm{O}$ & 6.5 & 6.2 & 6.2 \\
\hline $\mathrm{C} \mathrm{sp}$ & 8.8 & 15.8 & 14.9 \\
\hline $\mathrm{O}-\mathrm{C}=\mathrm{O}$ & 1.3 & 3.2 & 3.6 \\
\hline $\mathrm{C}-\mathrm{F}_{3}$ & - & 2.1 & - \\
\hline $\mathrm{C}-\mathrm{F}_{x}$ & - & 0.7 & - \\
\hline Total for carbon & 94.9 & 86.7 & 88.8 \\
\hline $\mathrm{N}$ 1s & 0.3 & 0.3 & 0.2 \\
\hline O $1 \mathrm{~s}$ & 4.8 & 8.5 & 10.7 \\
\hline F 1s & - & 3.5 & - \\
\hline Ag $3 d_{5 / 2}$ & - & 0.7 & - \\
\hline S 2p & - & 0.3 & 0.3 \\
\hline $\mathrm{Cl} 2 \mathrm{p}$ & - & 0.4 & - \\
\hline
\end{tabular}


Deconvolution of the high-resolution XPS spectrum of $\mathrm{C} 1 \mathrm{~s}$ spectrum (Fig. 2a) shows that MLG consists of seven environments for the carbon atoms. These were as follows with the binding energies within brackets: $\mathrm{C} \mathrm{sp}^{2}(284.5 \mathrm{eV}), \pi-\pi^{*}(290.9$ and $294.0 \mathrm{eV}), \mathrm{C}=\mathrm{O}(288.3 \mathrm{eV}), \mathrm{C}-\mathrm{O}(286.6 \mathrm{eV}), \mathrm{C} \mathrm{sp}^{2}(284.8 \mathrm{eV})$ and $\mathrm{O}-\mathrm{C}=\mathrm{O}(289.6 \mathrm{eV})$. A breakdown of the elemental compositions of these components is shown in Table 1. Carbons represent 94.9 at\% on the total elemental composition at the surface as expected from graphitic material.

The corresponding C 1s spectra for ${ }^{\mathrm{Ar}} f$-MLG (Fig. 2b), consists of nine components corresponding to $\mathrm{C} \mathrm{sp}^{2}(284.5 \mathrm{eV})$, $\pi-\pi^{*}(290.9$ and $294.0 \mathrm{eV}), \mathrm{C}=\mathrm{O}(287.7 \mathrm{eV}), \mathrm{C}-\mathrm{O}(286.4 \mathrm{eV})$, $\mathrm{C} \mathrm{sp}^{2}(284.8 \mathrm{eV}), \mathrm{O}-\mathrm{C}=\mathrm{O}(289.0 \mathrm{eV}), \mathrm{C}-\mathrm{F}_{x}(284.9 \mathrm{eV})$ and $\mathrm{CF}_{3}$ $(292.7 \mathrm{eV})$. Whilst both show graphitic character, ${ }^{\mathrm{Ar}} f$-MLG shows additional $\mathrm{C}-\mathrm{F}$ functionality, originating from the reaction of 4-(trifluoromethyl)phenyl radicals with the graphitic material. Overall, there is a reduction in the percentage carbon in this sample. This is consistent with functionalisation. Furthermore, there is a large increase in the concentration of $\mathrm{sp}^{3}$ hybridised carbon centres, with respect to $\mathrm{sp}^{2}$ hybridised carbons, from 8.8 at\% up to 15.8 at\%. Some of this increase has be attributed to oxidation of the MLG sample as a result of the oxidising conditions of the reaction (vide infra). As outlined below however, the level of increase in the oxygen content does not account for such a large increase in $\mathrm{sp}^{3}$ hybridised centres. These observations are therefore consistent with some degree of the covalent attachment of the aryl functional groups to the conjugated $\mathrm{sp}^{2}$ network to form ${ }^{\mathrm{Ar}} f$-MLG.

The $\mathrm{O}$ 1s spectrum for MLG (Fig. 3a) consists of five components $\mathrm{C}=\mathrm{O}(531.7 \mathrm{eV}), \mathrm{O} 1 \mathrm{~s} \mathrm{C}-\mathrm{O}-\mathrm{C}(533.1 \mathrm{eV})$ and $\mathrm{O} 1 \mathrm{~s}$ satellite structures ( $535.1 \mathrm{eV}, 536.9$ and $538.9 \mathrm{eV})$. This confirms the presence of a significant degree of oxygen functionality already within the starting material which originates from its plasma processing. The presence of these oxygen functionalities in MLG corresponds to 4.8 at\%. The $\mathrm{O}$ 1s spectra for ${ }^{\mathrm{Ar}} f$-MLG looks similar to MLG (Fig. 3b), displaying the same components corresponding to $\mathrm{C}=\mathrm{O}(531.7 \mathrm{eV}), \mathrm{C}-\mathrm{O}-\mathrm{C}(533.1 \mathrm{eV})$ and $O$ 1s satellite structures (535.1, 536.9 and $538.9 \mathrm{eV}$ ), with similar respective ratios. Upon functionalisation however, the oxygen content almost doubled, increasing from 4.8 to 8.5 at\%. This increase in oxygen content is attributed to the oxidising reagent which is required for radical generation. In addition to generating the 4-(trifluoromethyl)phenyl radical it also increased the level of oxidation of the graphene surface to some degree. In order to confirm this, we carried out a control reaction under the same conditions with only $\mathrm{K}_{2} \mathrm{~S}_{2} \mathrm{O}_{8}$ and a sample of MLG. We refer to this new material as ${ }^{\mathrm{c}} \mathrm{Ox}-\mathrm{MLG}$. Its surface elemental composition as determined by XPS is also presented in Table 1 for comparison. In this case, a large increase in the oxygen concentration was indeed observed in the sample up to 10.7 at\%. Even though the percentage of $\mathrm{sp}^{3}$ carbon centres in ${ }^{\mathrm{Ar}} f$-MLG is higher than ${ }^{\mathrm{c}} \mathrm{Ox}-\mathrm{MLG}$, the increase in oxygen content is much lower ( $c f .8 .5$ at\% vs. 10.7 at\%). This is consistent with the fact that the additional $\mathrm{sp}^{3}$ centres originate from aryl group functionalisation. Furthermore, an increase in oxygen content may also be explained by the fact that a portion of 4 -(trifluoromethyl)phenoxide $\left[\mathrm{OC}_{6} \mathrm{H}_{4}\left(\mathrm{CF}_{3}\right)\right]$ groups could also be covalently bonded to MLG in addition to $\mathrm{C}_{6} \mathrm{H}_{4}\left(\mathrm{CF}_{3}\right)$. The ether species (B) was of course found in the control reaction in the absence of MLG (where both A and $\mathbf{B}$ were formed).

Fig. 4 highlights the XPS spectra at higher binding energies, indicating the presence of other functional groups. Upon functionalisation, a peak at $687.9 \mathrm{eV}$ (Fig. 4a) confirms the presence of fluorine in the ${ }^{\mathrm{Ar}} f$-MLG material. This is not present within the starting material (see Fig. 1a and Table 1). This binding energy is consistent with the presence of the 4-(trifluoromethyl)phenyl functional group. ${ }^{36}$ Alongside this evidence of incorporation of this functional group are changes to the elemental compositions of carbon and oxygen. The carbon content reduced to 86.5 at\%, as expected. Furthermore, the scenario of physisorption of the starting material (trifluoromethylphenyl boronic acid) on to the MLG surface can be ruled out due to the absence of any boron content in the XPS data. The material was washed multiple times and the corresponding filtrates were monitored by ${ }^{19} \mathrm{~F}$ and ${ }^{1} \mathrm{H}$ NMR spectroscopy to ensure that the material was free of any unreacted or residual organic species.

The presence of silver ( $3 \mathrm{~d}$ orbitals) was observed in the spectrum for ${ }^{\mathrm{Ar}} f$-MLG at 367.0 and $372.8 \mathrm{eV}$. The former $\mathrm{Ag} 3 \mathrm{~d}_{5 / 2}$ peak was further deconvoluted revealing signals at $367.9 \mathrm{eV}$ and $366.9 \mathrm{eV}$ (Fig. 4b). These resemble silver salts in the form of $\mathrm{AgCl} / \mathrm{Ag}$ and $\mathrm{Ag}_{2} \mathrm{SO}_{4}$, respectively. ${ }^{37,38}$ This is likely to be due to silver salts retained within the material. These proved rather challenging to remove and they appeared to be trapped within the material despite multiple washings steps. Furthermore, both ${ }^{\mathrm{Ar}} \mathrm{f}$-MLG and ${ }^{\mathrm{c}} \mathrm{Ox}$-MLG materials revealed a small percentage of sulfur incorporation as a result of the oxidising agent. Again, these proved challenging to remove completely. Some nitrogen moieties are present within both MLG and ${ }^{\mathrm{Ar}} f$-MLG at relatively consistent compositions (see Fig. S4, ESI $\dagger$ and Table 1). The retention of these impurities within ${ }^{\mathrm{Ar}} f$-MLG results from the inherent nature of this plasmasynthesied graphitic material where the silver, for example, can get trapped within pores and defects in the material (vide infra).

\section{Determining the decomposition characteristics by TGA}

Thermogravimetric analysis (TGA) enables us to examine how the materials decompose, which can be very useful in confirming the nature and degree of functionalisation within the material. Accordingly, the thermal stability of both MLG and ${ }^{\mathrm{Ar}}$-MLG was investigated using TGA measurements at temperatures up to $700{ }^{\circ} \mathrm{C}$. A comparison of the results is depicted in Fig. 5. In both cases, a gradual mass loss is observed with distinct or well-defined mass loss regions. Pristine graphite has been found to show little decomposition until around $600{ }^{\circ} \mathrm{C}$ within an $\mathrm{O}_{2}$ atmosphere and up to $1000{ }^{\circ} \mathrm{C}$ within a nitrogen atmosphere. Accordingly, mass loss within both samples at these lower temperatures can be attributed to the decomposition of covalently and non-covalently bonded functionality over a gradual period. ${ }^{39,40}$ Initial mass loss (up to $100{ }^{\circ} \mathrm{C}$ ), is attributed to the removal of water from the surface. Decomposition from this 


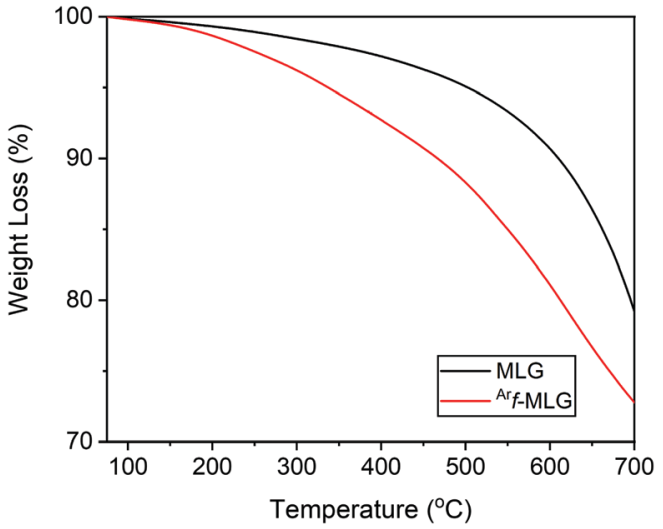

Fig. 5 TGA curves representing the thermal stability of MLG and ${ }^{A r} f-M L G$.

point is then assigned to the removal of covalently bonded oxygen functionality. In the case of graphene oxide, it has been found that the decomposition of covalently bound oxygen functionality takes place above $150{ }^{\circ} \mathrm{C} .{ }^{41 \mathrm{Ar}}$-MLG shows a lower thermally stability than MLG, decomposing at a faster rate. This is assigned to the increased covalent functionality it possesses, including more oxygen functionality and 4-(trifluoromethyl)phenyl moieties. The presence of silver salts on the surface of ${ }^{A r}$-MLG may also open up other pathways leading to a more rapid mass loss from this material.

\section{Examining the graphitic structure by Raman spectroscopy}

Raman spectroscopy was used to examine the graphitic structure and extent of defects within MLG and ${ }^{\mathrm{Ar}}$-MLG, using a wavelength of $514 \mathrm{~nm}$. Raman spectra for the functionalised and unfunctionalised material are depicted in Fig. 6. The spectra for the control material, ${ }^{\mathrm{c}} \mathrm{Ox}-\mathrm{MLG}\left(\mathrm{K}_{2} \mathrm{~S}_{2} \mathrm{O}_{8}\right.$ only) is presented in Fig. S3 (ESI $\dagger$ ). A comparison of the spectra for MLG and ${ }^{A r} f$-MLG revealed some changes to the structure of the two materials. Both contained the characteristic peaks for graphitic materials, most notably the $\mathrm{G}$ band which appeared at $1576.6 \mathrm{~cm}^{-1}$ for MLG and $1580.3 \mathrm{~cm}^{-1}$ for ${ }^{\mathrm{Ar}}$-MLG. This doubly

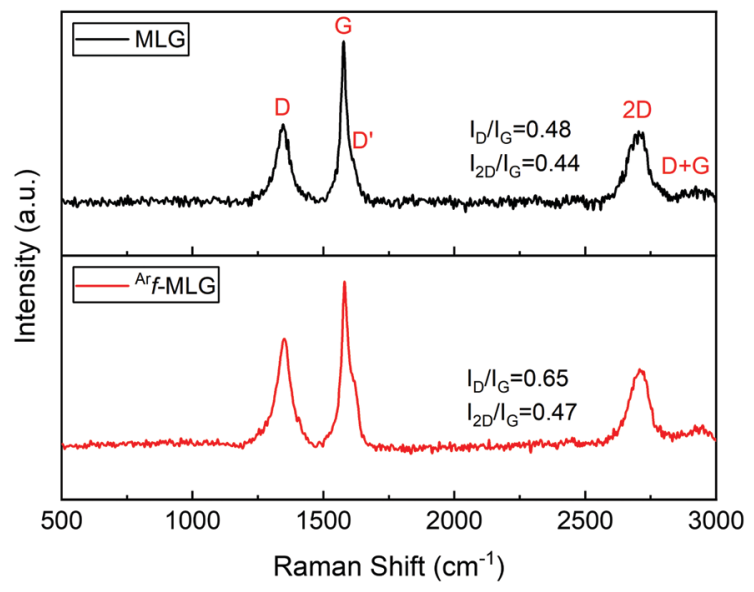

Fig. 6 Raman spectra of MLG (top) and ${ }^{A r} f-M L G$ (bottom). degenerate $\mathrm{E}_{2 \mathrm{~g}}$ band confirms the $\mathrm{sp}^{2}$ carbon network within the materials. ${ }^{10,42,43}$

The presence of strong $\mathrm{D}$ bands indicates the low crystallinity of the materials and also a high degree of $\mathrm{sp}^{3}$ carbon centres. This is expected for plasma-synthesised MLG's. The level of defects is revealed by the $\mathrm{D}$ bands, which occur at $1344.6 \mathrm{~cm}^{-1}$ (for MLG) and $1350.9 \mathrm{~cm}^{-1}$ (for ${ }^{\mathrm{Ar}} f-\mathrm{MLG}$ ). The D band represents the breathing modes with $A_{1 \mathrm{~g}}$ symmetry involving phonons near the K zone boundaries. ${ }^{44}$ The $2 \mathrm{D}$ bands at $2704.0 \mathrm{~cm}^{-1}$ (MLG) and $2708.0 \mathrm{~cm}^{-1}$ ( ${ }^{\mathrm{Ar}} f$-MLG) correspond to the second order symmetry allowed overtone of the $\mathrm{D}$ band. Within both spectra, the 2D bands are broad and heavily upshifted in respect to that of single layer graphene, suggesting the presence of multiple layered stacks. This is further confirmed by examining the relative intensity of the $\mathrm{G}$ band compared to the $2 \mathrm{D}$ band, thus providing $I_{2 \mathrm{D}} / I_{\mathrm{G}}$ ratios. ${ }^{45} I_{2 \mathrm{D}} / I_{\mathrm{G}}$ ratios lower than one are indicative of multi-layered structures. ${ }^{46}$ The ratios for MLG and ${ }^{A r}$-MLG are 0.44 and 0.47 , respectively. Additional defect-induced bands, which are more intense in the spectrum for ${ }^{\mathrm{Ar}}$-MLG, are observed at $1618 \mathrm{~cm}^{-1}$ and $2930 \mathrm{~cm}^{-1} .47,48$

The higher level of defects within the MLG material most likely originates from the plasma processing during the synthesis. This can lead to an increased number of covalently attached oxygen functionalities at the outer surfaces of the sheets. Addition of these oxygen groups to the $\mathrm{sp}^{2}$ carbons within the material increases the number of sites of $\mathrm{sp}^{3}$ hybridised carbon atoms. The intensity ratio of the $\mathrm{D}$ band in relation to the $\mathrm{G}$ band $\left(I_{\mathrm{D}} / I_{\mathrm{G}}\right)$, is indicative of the degree of functionalisation. The estimated $I_{\mathrm{D}} / I_{\mathrm{G}}$ ratios for MLG and ${ }^{\mathrm{Ar}}$-MLG are 0.48 and 0.65 , respectively. The larger ratio for ${ }^{\mathrm{Ar}}$-MLG indicates an increase in $\mathrm{sp}^{3}$ sites, and thus, this confirms an increase in the covalent functionalisation in comparison to the original MLG material. This increase is likely to originate from the desired 4-(trifluoromethyl)phenyl incorporation and additional oxygen functionality. As highlighted above, the XPS analysis indeed confirmed a significant increase in oxygen functionality which was attributed to the oxidising agent $\mathrm{K}_{2} \mathrm{~S}_{2} \mathrm{O}_{8}$. In order to confirm this, the Raman spectrum of ${ }^{\mathrm{C} O x-M L G}$ was also examined. This exhibited similar changes to those found in ${ }^{\mathrm{Ar}} f$-MLG, however, to a lesser extent with respect to the starting MLG. For example, the $I_{\mathrm{D}} / I_{\mathrm{G}}$ and $I_{2 \mathrm{D}} / I_{\mathrm{G}}$ ratios were found to be 0.56 and 0.40 ( $c f$. with the values 0.65 and 0.47 for ${ }^{\mathrm{Ar}} f-\mathrm{MLG}$ ). This is consistent with the incorporation of 4-(trifluoromethyl)phenyl groups onto the MLG material alongside some increase in the oxidation level of the material. It should be noted that the level of functionalisation is estimated to be lower than some diazonium salt methodologies and thus further optimisation of this new strategy is needed. Nevertheless, both the Raman spectroscopic and XPS data are both consistent with functionalisation of MLG with trifluoromethylphenyl groups.

\section{Analysis of interlayer spacing using X-ray diffraction data}

In order to gain more detailed information on the interlayer spacing and orientation of the planes within MLG and ${ }^{\mathrm{Ar}} f$-MLG, an X-ray diffraction (XRD) investigation was carried out. The XRD 
spectra for both MLG and ${ }^{\mathrm{Ar}}$-MLG are shown in Fig. 7. The spectra show that both materials contain hexagonal $\mathrm{ABAB}$ stacking $(2 \mathrm{H})$ and rhombohedral ABCA (3R) stacking. This is consistent with that found in commercially available graphene and graphitic-based materials. ${ }^{49}$ Strong diffraction peaks are present within both spectra at $26.6^{\circ}$, corresponding to graphitic $2 \mathrm{H}(002)$ and $3 \mathrm{R}$ (003) planes, with an interlaying spacing of $3.35 \AA$. Analysis of the line shape of this signal suggests that for the majority of the materials, the number of graphene layers within both MLG and ${ }^{\mathrm{Ar}} f$-MLG are in the region of 58 to 73 layers (see ESI $\dagger$ for further details). As such, the material could therefore also be described as graphite nanostructures. ${ }^{50}$ For MLG, two smaller intensity lines are also observed at $42.59^{\circ}$ and $44.56^{\circ}$, which correspond to the $2 \mathrm{H},(100)$ and (101) stacking planes. Two further small intensity lines are observed at $43.44^{\circ}$ and $46.20^{\circ}$, which correspond to the $3 \mathrm{R},(101)$ and (012) stacking planes. The presence of this four-lined pattern is more clearly seen in the expanded section of Fig. 7. This provides evidence of the $3 \mathrm{R}$ and $2 \mathrm{H}$ phases within the structure. For these, a ratio of $49: 51 \%(3 \mathrm{R}: 2 \mathrm{H})$ was determined (see $\mathrm{ESI} \dagger$ for details). These are consistent with that observed within various related materials. ${ }^{49,51}$ Again, for MLG two additional lines at $54.73^{\circ}$ and $77.62^{\circ}$ are observed which correspond to graphite $2 \mathrm{H}(004)$ and (110) planes.

Many additional diffraction lines are present within the spectrum of the ${ }^{\mathrm{Ar}} f$-MLG sample, suggesting other chemical species have been incorporated within the structure. The characteristic four-line pattern also appears to be present within this spectrum albeit the $2 \mathrm{H}(101)$ and $3 \mathrm{R}$ (012) lines are obscured by some new lines. This confirms that there is no significant change to the interlayer spacing upon functionalisation. As highlighted with asterisks $\left(^{*}\right)$, the majority of these correspond to the diffraction of the $\mathrm{AgCl}, \mathrm{Ag}$ and $\mathrm{Ag}_{2} \mathrm{SO}_{4}$ impurities incorporated into the material (PDF card numbers: $00-006-0480,{ }^{52} 01-073-6977^{53}$ and $\left.01-074-1739^{54}\right)$. The presence of these silver compounds is in agreement with XPS data presented above. There are no observable lines at $2 \theta$ angles lower that $26.6^{\circ}$ This suggests that largest interlayer spacing
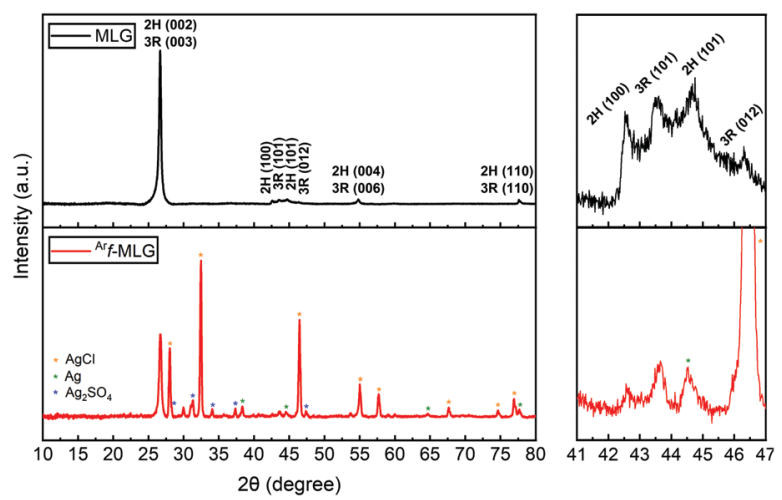

Fig. 7 XRD spectra of MLG (top) and ${ }^{A r} f-M L G$ (bottom) in the range between $2 \theta=10^{\circ}$ and $80^{\circ}$ (left) and expanded section in the range between $2 \theta=41^{\circ}$ and $47^{\circ}$ (right). The * denote lines associated with silver compounds (see text for details) value between planes corresponds to the graphitic planes. Therefore, it is most likely that functionalisation has taken place at the outer surfaces of the material. Functionalisation within the internal MLG structure would, of course, result in an increase in the interlayer spacing. The lines corresponding to the $2 \mathrm{H}$ and $3 \mathrm{R}$ arrangements in both MLG and ${ }^{\mathrm{Ar}} f$-MLG show only minor differences confirming the functionalisation has only a small impact on the crystallinity and interlayer spacing of the MLG structure. Again, this is consistent with functionalisation at the outer positions only.

\section{Analysis of surface morphology using SEM and TEM}

Scanning electron microscopy (SEM) and transmission electron microscopy (TEM) were used to investigate the surface morphology and lateral dimensions of the graphitic sheets. Four SEM images were taken of a representative example of each material at low and high magnifications (Fig. 8 and 9). The images reveal that both materials consist of multi particulate material as agglomerates which range in diameter between 10-30 $\mu \mathrm{m}$ for MLG and $<10 \mu \mathrm{m}$ in size for ${ }^{\mathrm{Ar}} f$-MLG. The quantity of agglomerates within MLG exceeds that of ${ }^{\mathrm{Ar}} f$-MLG, suggesting that the functionalisation process has led these loosely bound aggregates to unravel. At lower magnifications MLG and ${ }^{A r} f$-MLG display a powder like appearance. At increased magnifications, it can be seen that these agglomerates are irregularly arranged consisting of flakes comprised of mostly multi-layered stacks, physically aggregated by $\pi-\pi$ interactions. These stacks adopt wavy topography and curled edges. The non-uniform nature of the morphology of these materials is most evident in the higher resolution images. These defects are likely to result from the plasma ablation process during synthesis. The particle size distribution is due to the method of processing of this material.

Under the conditions utilised to record the images, it was found that the samples underwent charging. In order to obtain more enhanced images, gold coating was added using a sputter coater. The gold coating can be seen with a scaly appearance, as exemplified in Fig. 10.

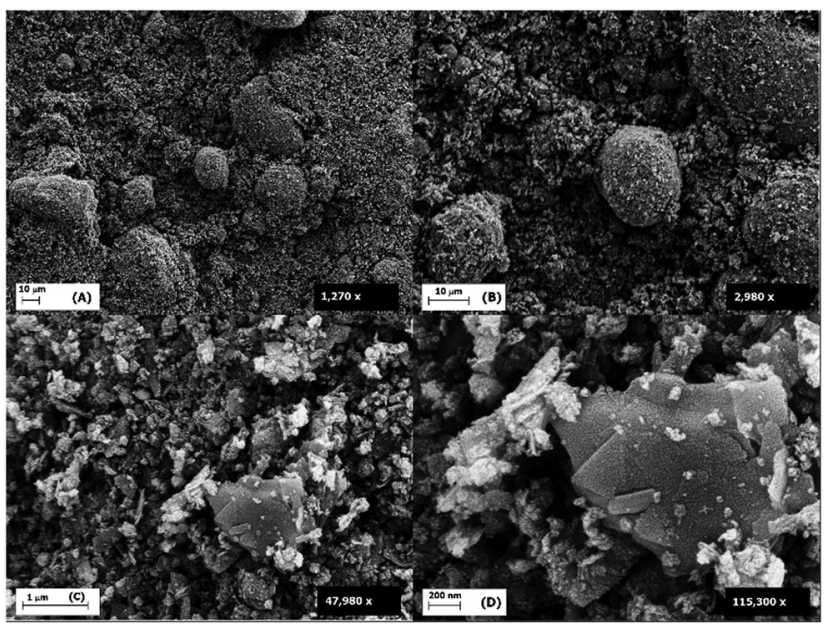

Fig. 8 Representative SEM images for MLG at low and high magnifications. 


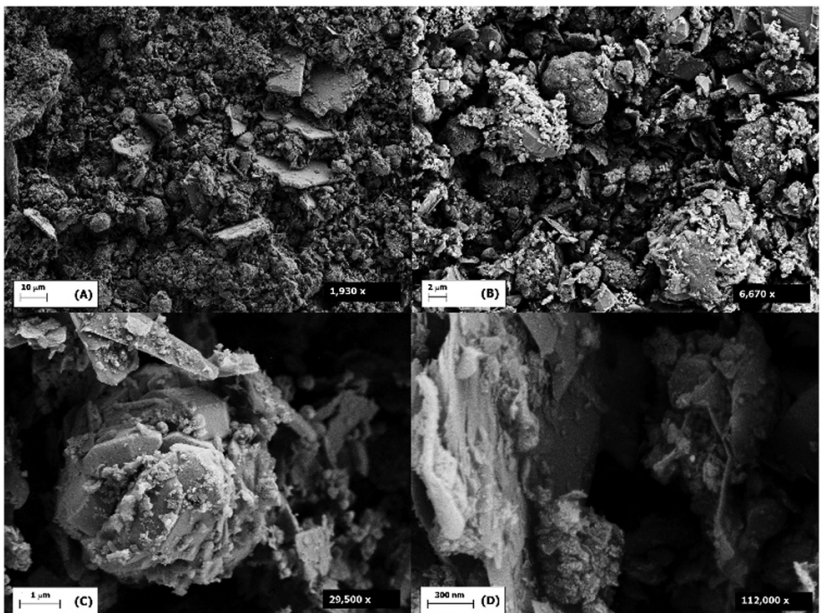

Fig. 9 Representative SEM images for ${ }^{A r} f-M L G$ at low and high magnifications

Additional SEM images of ${ }^{A r} f$-MLG are provided in Fig. 11. These highlight some of the features of the materials related to their porosity. As can be seen in the image on the left, there is a macro sized pore (circled) which contains some additional material. We believe that this could be some smaller flakes of ${ }^{\mathrm{Ar}} f$-MLG which become trapped within the in-plane pore, and for this reason are difficult to remove during the purification process. The presence of material which could be trapped silver nanomaterials can also be seen on an image in Fig. S7 (see ESI $\dagger$ ). The image on the right of Fig. 11 highlights the slit like channels which are found within the spacing between subsequent stacks. The implications of these features on the surface area and porosity of the MLG and ${ }^{\mathrm{Ar}} f$-MLG materials are discussed in the following section.

Selected TEM images of MLG and ${ }^{\mathrm{Ar}}$-MLG are presented in Fig. 12. These also shown that both materials consist of multiple platelets with significant clumping. These multi-layered structures can be observed by differences in the contrast. Darker areas within the TEM images represent more dense areas within increased number of layers, whilst light areas represent those of less layers. It can be observed by TEM images that the stacks of layers exhibit lateral dimensions between 100-500 $\mathrm{nm}$ in diameter. As with the SEM images, they also indicate material which contains a broad range of morphologies across the surface of both materials.

When comparing the SEM and TEM images for ${ }^{\mathrm{Ar}} f$-MLG with those of MLG, it can be concluded that there are no visual

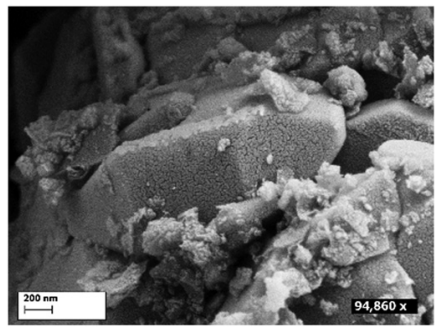

Fig. 10 SEM image for ${ }^{A r} f$-MLG highlighting the scaly appearance.

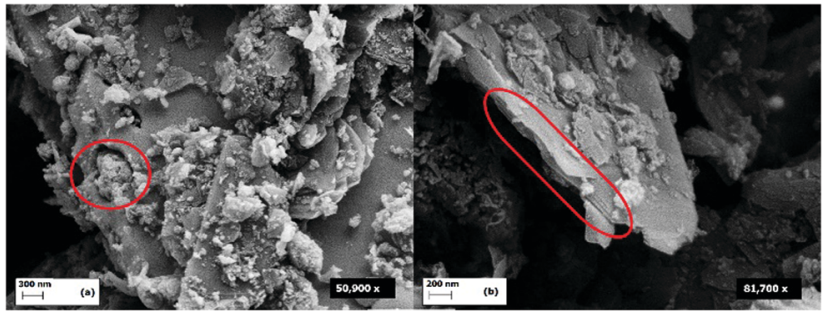

Fig. 11 SEM images of ${ }^{A r}$-MLG showing; a blocked macropore (left) and slit like channels (right).

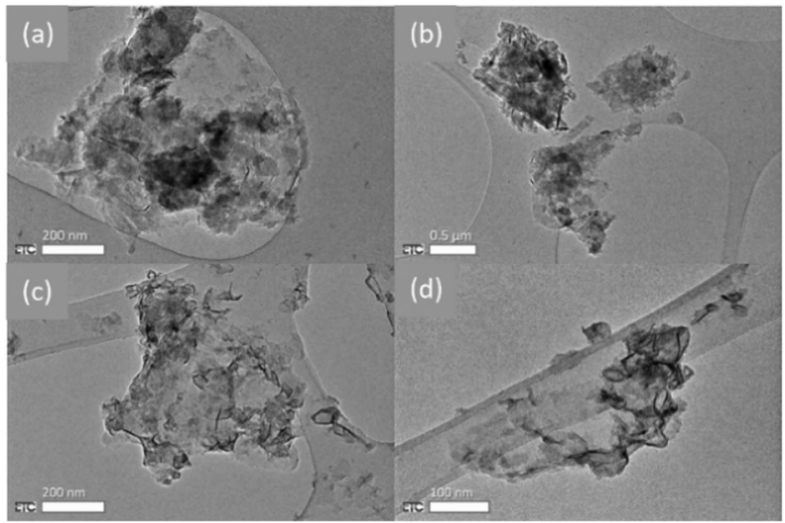

Fig. 12 TEM images for MLG $(a+b)$ and ${ }^{A r} f-M L G(c+d)$.

changes of major significance in the morphology upon functionalisation. Nevertheless, the orientation of stacks in ${ }^{\mathrm{Ar}}$-MLG appear to be less agglomerated than those within MLG. This causes the individual stacks to appear much clearer within the SEM images. TEM images also reveal that the flakes remain very similar in size and distribution.

\section{Surface area and porosity analysis}

Nitrogen adsorption-desorption measurements were used to determine the surface area and pore size distribution of MLG
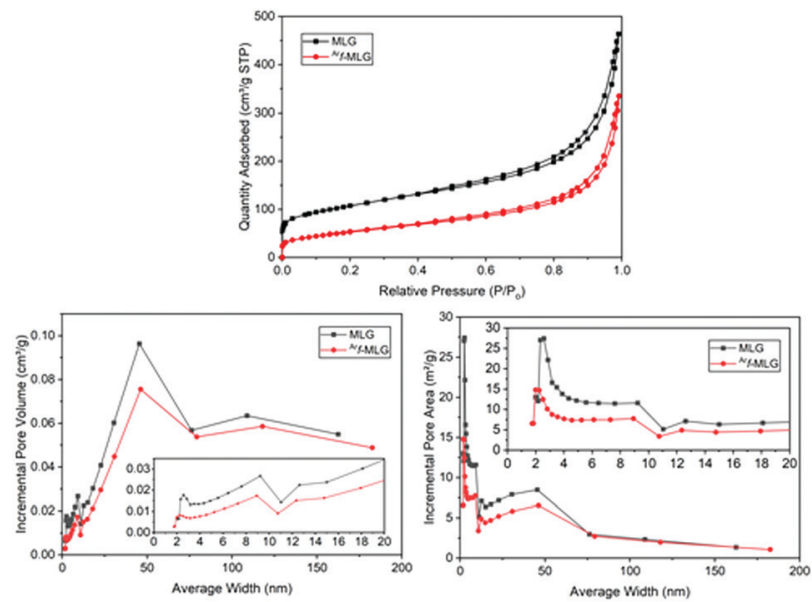

Fig. 13 BET data for MLG and ${ }^{A} f$-MLG including: (a) $N_{2}$ adsorptiondesorption isotherm (b) pore size distribution as a function of incremental pore volume (c) pore size distribution as a function of incremental pore area. 
Table 2 BET surface area, BJH adsorption cumulative surface area of pores between 1.7-300 nm and BJH adsorption average pore width data for MLG and ${ }^{\operatorname{Ar}} f-M L G$

\begin{tabular}{|c|c|c|}
\hline & MLG & ${ }^{\mathrm{Ar}} f$-MLG \\
\hline $\mathrm{BJH}$ adsorption cumulative surface area of pores between $1.7-300 \mathrm{~nm}\left(\mathrm{~m}^{2} \mathrm{~g}^{-1}\right)$ & 287.01 & 167.86 \\
\hline Percentage contribution of pores between $1.7-300 \mathrm{~nm}$ to the total BET surface area (\%) & 75.50 & 87.40 \\
\hline
\end{tabular}

and ${ }^{\mathrm{Ar}}$-MLG, utilising Branauer-Emmett-Teller (BET) analysis and Barret-Joyner-Halenda (BJH) analysis. ${ }^{55}$ The results of these investigations are presented in Fig. 13. It was found that MLG and ${ }^{\text {Ar }}$-MLG both exhibited Type IIb $\mathrm{N}_{2}$ adsorptiondesorption isotherms typical of materials composed of platelike particles (Fig. 13a) ${ }^{56}$ A Type H3 hysteresis loop is present in each isotherm, indicating the occurrence of capillary condensation within pores of $\sim 4 \mathrm{~nm}$ in size. ${ }^{57}$ Hysteresis of this type is usually associated with plate-like aggregates or adsorbents containing slit-like pores.

MLG and ${ }^{\mathrm{Ar}}$-MLG were found to exhibit BET surface areas corresponding to 380.22 and $192.13 \mathrm{~m}^{2} \mathrm{~g}^{-1}$, respectively (Table 2). A significant contribution of this area originates from pores between 1.7-300 $\mathrm{nm}$. These pores are mostly present within the space between neighbouring stacks which correspond to $75.5 \%$ (MLG) and $87.4 \%{ }^{\mathrm{Ar}}$-MLG of the total surface area.

The pore size distribution profile was examined by plotting the pore size distribution as a function of incremental pore volume (Fig. 13b) and incremental pore area using the adsorption branch of the isotherm (Fig. 13c). Overall, the curves reveal similar patterns for both MLG and ${ }^{\mathrm{Ar}}$-MLG in each plot, suggesting a minor impact to the overall structure upon functionalisation. In parallel, the average pore size for MLG and ${ }^{A r}$-MLG were $9.53 \mathrm{~nm}$ and $11.70 \mathrm{~nm}$, respectively. The main difference, however, is a decrease in the area and volume of pores affecting mostly pores with diameters of less than $\sim 75 \mathrm{~nm}$. This is an indication that functionalisation causes a significant reduction in the quantity of pores within the mesoporous region. This finding coincides with the significant decrease in BET surface area (almost half of MLG). We suggest that the functionalised sheets re-orientate themselves to adopt curled, scroll-like edges due to enhanced interactions between the functionalised stacks.

With such a situation, many of the slit-like pores and channels between neighbouring stacks would become inaccessible to the nitrogen adsorbent molecules. In addition, upon functionalisation, we also observe blockage of pores with smaller aggregates (as seen in Fig. 11). This is can be clearly seen in the SEM image in Fig. 11a which shows a macropore with a width of $700 \mathrm{~nm}$ in addition to Fig. 11b where stacks of sheets can be observed. ${ }^{58}$

\section{Experimental}

\section{General remarks}

All solvents and reagents were purchased from commercial suppliers and used with no further purification. The MLG material was synthesised and provided by Perpetuus Carbon Technologies.
Synthesis of MLG. Natural flake plasma processing of natural graphite was carried out using a custom-made multi-electrode dielectric barrier discharge (DBD) plasma reactor as described elsewhere. ${ }^{29}$

Control reaction: reactivity of radicals generated from 4-(trifluoromethyl)phenyl boronic acid in the absence of MLG. This reaction was carried out using similar conditions to that of previous works. ${ }^{32}$ The reagent 4 -(trifluoromethyl)phenyl boronic acid $\left(0.092 \mathrm{~g}, 4.83 \times 10^{-4} \mathrm{~mol}\right)$ was dissolved in a solvent mixture $(40 \mathrm{~mL})$ consisting of water and DCM $(1: 1)$, followed by the addition of $\mathrm{K}_{2} \mathrm{~S}_{2} \mathrm{O}_{8}\left(0.266 \mathrm{~g}, 9.85 \times 10^{-4} \mathrm{~mol}\right)$ and $\mathrm{AgNO}_{3}$ $\left(0.027 \mathrm{~g}, 1.60 \times 10^{-4} \mathrm{~mol}\right)$. The mixture was allowed to stir for $44 \mathrm{~h}$ at room temperature. The biphasic mixture was filtered and the DCM layer was separated from the filtrate using a separating funnel. The DCM layer was then evaporated to dryness to give a yellow oil consisting of two compounds: 4,4'-bis(trifluoromethyl)biphenyl (A) and bis((trifluoromethyl)diphenyl)ether (B). Characterisation of $\mathbf{A}:{ }^{1} \mathrm{H}$ NMR $\delta\left(\mathrm{CD}_{3} \mathrm{CN}\right)$ : $7.87\left(\mathrm{~d},{ }^{3} J_{\mathrm{HH}}=8.2 \mathrm{~Hz}, 4 \mathrm{H}\right), 7.81\left(\mathrm{~d},{ }^{3} J_{\mathrm{HH}}=8.2 \mathrm{~Hz}, 4 \mathrm{H}\right) .{ }^{19} \mathrm{~F} \mathrm{NMR}$ $\delta\left(\mathrm{CD}_{3} \mathrm{CN}\right):-63.06$. EI-MS $m / z[\mathrm{M}]^{+}$calc. for $\mathrm{C}_{14} \mathrm{H}_{8} \mathrm{~F}_{6} 290$ Da: found: $290 \mathrm{Da}$. Characterisation of $\mathbf{B}:{ }^{1} \mathrm{H}$ NMR $\delta\left(\mathrm{CD}_{3} \mathrm{CN}\right): 7.72$ $\left(\mathrm{d},{ }^{3} \mathrm{~J}_{\mathrm{HH}}=8.2 \mathrm{~Hz}, 4 \mathrm{H}\right), 7.20\left(\mathrm{~d},{ }^{3} \mathrm{~J}_{\mathrm{HH}}=8.2 \mathrm{~Hz}, 4 \mathrm{H}\right) .{ }^{19} \mathrm{~F} \mathrm{NMR}$ $\delta\left(\mathrm{CD}_{3} \mathrm{CN}\right):-62.42$. EI-MS $\mathrm{m} / z[\mathrm{M}]^{+}$calc. for $\mathrm{C}_{14} \mathrm{H}_{8} \mathrm{~F}_{6} \mathrm{O} 306 \mathrm{Da}$ found $306 \mathrm{Da}$.

Synthesis of ${ }^{\mathrm{Ar}} \boldsymbol{f}$-MLG. 4-(Trifluoromethyl)phenyl boronic acid $\left(0.930 \mathrm{~g}, 4.91 \times 10^{-3} \mathrm{~mol}\right)$ was added to MLG $(0.720 \mathrm{~g})$ and dispersed in a solvent mixture $(40 \mathrm{~mL})$ consisting of water and DCM (1:1) followed by the addition of $\mathrm{K}_{2} \mathrm{~S}_{2} \mathrm{O}_{8}(2.600 \mathrm{~g}$, $\left.9.62 \times 10^{-3} \mathrm{~mol}\right)$ and $\mathrm{AgNO}_{3}\left(1.180 \mathrm{~g}, 7.00 \times 10^{-3} \mathrm{~mol}\right)$. The mixture was allowed to stir for $44 \mathrm{~h}$ at room temperature. The reaction mixture was then centrifuged and the resultant ${ }^{\mathrm{Ar}}$-MLG solid was separated and washed repeatedly with water, acetonitrile, and DCM through several dispersion/centrifugation cycles (4000 rpm, $20 \mathrm{~min}$ ). The resultant ${ }^{\mathrm{Ar}} f$-MLG was dried under vacuum $\left(10^{-6}\right.$ bar) for 1 week, to remove any residual solvents and traces of volatile components $\mathbf{A}$ and $\mathbf{B}$.

Control reaction: MLG treated with potassium persulfate ('Ox-MLG). MLG $(0.160 \mathrm{~g})$ and $\mathrm{K}_{2} \mathrm{~S}_{2} \mathrm{O}_{8}\left(0.570 \mathrm{~g}, 2.120 \times 10^{-3} \mathrm{~mol}\right)$ were dispersed in a solvent mixture $(20 \mathrm{~mL})$ consisting of water and DCM (1:1). The mixture was allowed to stir for $44 \mathrm{~h}$ at room temperature. The reaction mixture was then centrifuged and the resultant ${ }^{\mathrm{c}} \mathrm{Ox}$-MLG solid was separated and washed repeatedly with water, acetonitrile, and DCM through several dispersion/centrifugation cycles. The resultant material was dried in vacuum for 1 week.

\section{Characterisation methods}

X-Ray photoelectron spectroscopy (XPS) analysis was performed using a Kratos Axis Ultra-DLD photoelectron spectrometer with 
a monochromatic $\mathrm{Al} \mathrm{K}$ electron source (1486.6 eV). XPS data was analysed using CasaXPS and binding energies were referenced to carbon core level for adventitious carbon at $284.8 \mathrm{eV}$ possessing an uncertainty of $\sim 0.2 \mathrm{eV}$. Curve fitting was carried out using Gaussian and Lorentzian line profiles.

Thermal gravimetric analysis (TGA) was carried out using a PerkinElmer TGA 4000 instrument. The samples were heated from room temperature up to $900{ }^{\circ} \mathrm{C}\left(5{ }^{\circ} \mathrm{C} \mathrm{min}^{-1}\right)$ under a nitrogen atmosphere $\left(50 \mathrm{~mL} \mathrm{~min}^{-1}\right)$.

Raman spectroscopy was performed using a Renishaw inVia confocal Raman microscope equipped with an $\mathrm{Ar}^{+}$visible green laser with an emission wavelength of $514 \mathrm{~nm}$. Spectra were collected in a reflective mode by a high sensitive charge couple device (CCD) detector.

Powder X-ray diffraction (XRD) patterns were collected using a Panalytical X'Pert diffractometer with a $\mathrm{Cu}$ anode irradiation $(\lambda=1.541 \AA)$ operating at $40 \mathrm{kV}$ and $40 \mathrm{~mA}$. Phase identification was performed by matching experimental patterns against entries in the ICDD standard database.

Scanning electron microscopy (SEM) images were obtained using a Zeiss Supra 35VP (FEG) SEM instrument. The samples were gold-coated using a sputtering coater to enhance the resolution of the images.

Transmission electron microscopy (TEM) images were obtained using a Jeol 2100 field emission gun (FEG) TEM with a $200 \mathrm{kV}$ power source.

The surface area and porosity characteristics of the materials were analysed using a Micromeritics ASAP 2020 physisorption analyser. Samples were degassed under $0.667 \mathrm{~Pa}$ for 720 minutes at $150{ }^{\circ} \mathrm{C}$ with a heating rate of $10 \mathrm{~min}^{-1}$. The surface area and pore size distribution were measured at $77 \mathrm{~K}$ using Brunauer Emmett Teller (BET) and Barrett Joyner Halenda $(\mathrm{BJH})$ cumulative pore volume methods, respectively.

${ }^{1} \mathrm{H}$ and ${ }^{19} \mathrm{~F}$ NMR spectra were performed on a Bruker $400 \mathrm{MHz}$ Ascend $^{\mathrm{TM}} 400$, which operated at $400 \mathrm{MHz}$ for ${ }^{1} \mathrm{H}$ nuclei and 376.6 $\mathrm{MHz}$ for ${ }^{19} \mathrm{~F}$ nuclei. Chemical shifts are reported in parts per million (ppm). NMR spectra were obtained in $\mathrm{CD}_{3} \mathrm{CN}$ solvent (1.93 ppm) and internal reference for ${ }^{19} \mathrm{~F}$.

Mass spectrometry (MS) was carried out on compounds A and B using a Thermoscientific ISQ Single quad with direct insertion probe and the identity of the compounds were confirmed for the preinstalled library of compounds.

\section{Conclusions}

In this article, we provide a preliminary account outlining the successful covalent functionalisation of MLG with 4-(trifluoromethyl)phenyl radicals. This has been achieved by using 4-(trifluoromethyl)phenyl boronic acid as a radical source utilising Baran's protocol. The newly formed material, ${ }^{\mathrm{Ar}} f$-MLG, was found to be decorated at a number of positions at the outer surface as confirmed by a number of spectroscopic and analytical techniques.

At this stage of the development, some challenges associated with this functionalisation methodology and the nature of the plasma-synthesised multi-layer graphitic material have been identified. The attachment of 4-(trifluoromethyl)phenyl moieties is accompanied by an increase in oxygen functionality around the outer surfaces of the MLG stacks, as a result of the oxidising conditions. Furthermore, the task of removal of entrapped impurities, particularly silver in this case, will need to be addressed. Nevertheless, early indications suggest that this approach could provide access to aryl radicals in a costeffective and safer alternative to hazardous diazonium salts. As a result, this methodology could offer a novel safer approach to synthesise functionalised MLG materials on a larger scale with potential to be developed industrially. We are currently investigating ways in which this methodology can be optimised for practical application and exploring other derivatives. This may assist further processing and provide enhanced interaction with other materials. The development of new functionalisation strategies on commercially derived graphitic materials, of course, becomes increasingly important applications across materials science.

\section{Conflicts of interest}

There are no conflicts to declare.

\section{Acknowledgements}

This work was funded by European Social Fund (ESF) via the Welsh Government through a KESS2 PhD studentship for R. M. It was co-sponsored by Perpetuus Carbon Technologies. The company is also thanked for providing the raw MLG material. We are also grateful to Dr Gregg Shaw (Cardiff University) and the HarwellXPS EPSRC National Facility for X-Ray Photoelectron Spectroscopy Service for carrying out the Raman, XPS and TGA data collections and helpful discussions. We are additionally grateful to Dr Uchechukwu K. Onwukwe, Dr Ashley Howkins and Dr Lorna Anguilano at the Experimental Techniques Centre, Brunel University London, for running SEM and TEM analysis.

\section{References}

1 K. S. Novoselov, A. K. Geim, S. V. Morozov, D. Jiang, Y. Zhang, S. V. Dubonos, I. V. Grigorieva and A. A. Firsov, Electric Field Effect in Atomically Thin Carbon Films, Science, 2004, 306, 666-669.

2 Y. Zhu, H. Ji, H.-M. Cheng and R. S. Ruoff, Mass production and industrial applications of graphene materials, Natl. Sci. Rev., 2018, 5, 90-101.

3 P. Bazylewski and G. Fanchini, Comprehensive Nanoscience and Nanotechnology, Elsevier, 2019, vol. 1, pp. 287-304.

4 L. Lin, H. Peng and Z. Liu, Synthesis challenges for graphene industry, Nat. Mater., 2019, 18, 520-524.

5 D. W. Johnson, B. P. Dobson and K. S. Coleman, A manufacturing perspective on graphene dispersions, Curr. Opin. Colloid Interface Sci., 2015, 20, 367-382. 
6 A. P. Kauling, A. T. Seefeldt, D. P. Pisoni, R. C. Pradeep, R. Bentini, R. V. B. Oliveira, K. S. Novoselov and A. H. Castro Neto, The Worldwide Graphene Flake Production, Adv. Mater., 2018, 30, 1-6.

7 V. Georgakilas, M. Otyepka, A. B. Bourlinos, V. Chandra, N. Kim, K. C. Kemp, P. Hobza, R. Zboril and K. S. Kim, Functionalization of Graphene: Covalent and Non-Covalent Approaches, Derivatives and Applications, Chem. Rev., 2012, 112, 6156-6214.

8 G. H. Yang, D. D. Bao, H. Liu, D. Q. Zhang, N. Wang and H. T. Li, Functionalization of Graphene and Applications of the Deriviatives, J. Inorg. Organomet. Polym., 2017, 27, 1129-1141.

9 B. J. Hong, O. C. Compton, Z. An, I. Eryazici and S. T. Nguyen, Successful Stabilization of Graphene Oxide in Electrolyte Solutions: Enhancement of Biofunctionalization and Cellular Uptake, ACS Nano, 2012, 6, 63-73.

10 S. Niyogi, E. Bekyarova, M. E. Itkis, H. Zhang, K. Shepperd, J. Hicks, M. Sprinkle, C. Berger, C. N. Lau, W. A. DeHeer, E. H. Conrad and R. C. Haddon, Spectroscopy of Covalently Functionalized Graphene, Nano Lett., 2010, 10, 4061-4066.

11 J. M. Englert, C. Dotzer, G. Yang, M. Schmid, C. Papp, J. M. Gottfried, H.-P. Steinrück, E. Spiecker, F. Hauke and A. Hirsch, Covalent bulk functionalization of graphene, Nat. Chem., 2011, 3, 279-286.

12 H. Liu, S. Ryu, Z. Chen, M. L. Steigerwald, C. Nuckolls and L. E. Brus, Photochemical Reactivity of Graphene, J. Am. Chem. Soc., 2009, 131, 17099-17101.

13 C. K. Chua and M. Pumera, Friedel-Crafts Acylation on Graphene, Chem. - Asian J., 2012, 7, 1009-1012.

14 T. A. Strom, E. P. Dillon, C. E. Hamilton and A. R. Barron, Nitrene addition to exfoliated graphene: a one-step route to highly functionalized graphene, Chem. Commun., 2010, 46, 4097-4099.

15 S. Sarkar, E. Bekyarova, S. Niyogi and R. C. Haddon, DielsAlder chemistry of graphite and graphene: graphene as diene and dienophile, J. Am. Chem. Soc., 2011, 133, 3324-3327.

$16 \mathrm{H}$. Xu and K. S. Suslick, Sonochemical Preparation of Functionalized Graphenes, J. Am. Chem. Soc., 2011, 133, 9148-9151.

17 H. Ismaili, D. Geng, A. X. Sun, T. T. Kantzas and M. S. Workentin, Light-Activated Covalent Formation of Gold Nanoparticle-Graphene and Gold Nanoparticle-Glass Composites, Langmuir, 2011, 27, 13261-13268.

18 X. Zhong, J. Jin, S. Li, Z. Niu, W. Hu, R. Li and J. Ma, Arynecycloaddition: highly efficient chemical modification of graphene, Chem. Commun., 2010, 46, 7340-7342.

19 S. Mahouche-Chergui, S. Gam-Derouich, C. Mangeney and M. M. Chehimi, Aryl diazonium salts: a new class of coupling agents for bonding polymers, biomacromolecules and nanoparticles to surfaces, Chem. Soc. Rev., 2011, 40, 4143-4166.

20 G. L. C. Paulus, Q. H. Wang and M. S. Strano, Covalent Electron Transfer Chemistry of Graphene with Diazonium Salts, Acc. Chem. Res., 2013, 46, 160-170.

21 P. Huang, H. Zhu, L. Jing, Y. Zhao and X. Gao, Graphene Covalently Binding Aryl Groups: Conductivity Increases Rather than Decreases, ACS Nano, 2011, 5, 7945-7949.
22 A. Wang, W. Yu, Z. Huang, F. Zhou, J. Song, Y. Song, L. Long, M. P. Cifuentes, M. G. Humphrey, L. Zhang, J. Shao and C. Zhang, Covalent functionalization of reduced graphene oxide with porphyrin by means of diazonium chemistry for nonlinear optical performance, Sci. Rep., 2016, 6, 23325, DOI: 10.1038/srep23325.

23 E. Bekyarova, M. E. Itkis, P. Ramesh, C. Berger, M. Sprinkle, W. A. de Heer and R. C. Haddon, Chemical Modification of Epitaxial Graphene: Spontaneous Grafting of Aryl Groups, J. Am. Chem. Soc., 2009, 131, 1336-1337.

24 R. Sharma, J. H. Baik, C. J. Perera and M. S. Strano, Anomalously large reactivity of single graphene layers and edges toward electron transfer chemistries, Nano Lett., 2010, 10, 398-405.

25 Z. Xia, F. Leonardi, M. Gobbi, Y. Liu, V. Bellani, A. Liscio, A. Kovtun, R. Li, X. Feng, E. Orgiu, P. Samorì, E. Treossi and V. Palermo, Electrochemical Functionalization of Graphene at the Nanoscale with Self-Assembling Diazonium Salts, ACS Nano, 2016, 10, 7125-7134.

26 C. Peng, Y. Xiong, Z. Liu, F. Zhang, E. Ou, J. Qian, Y. Xiong and $\mathrm{W}$. Xu, Bulk functionalization of graphene using diazonium compounds and amide reaction, Appl. Surf. Sci., 2013, 280, 914-919.

27 S. Rooyanian, M. Bagherzadeh, Z. Akrami and A. N. Golikand, A simple route to surface functionalization of graphene nanosheets by benzoic acid and its application toward Pb(ii) sensing, New J. Chem., 2018, 42, 17371-17378.

28 M. Sheng, D. Frurip, D. Gorman and J. Loss, Reactive chemical hazards of diazonium salts, Prev. Process Ind., 2015, 38, 114-118.

29 I. Walters and D. Walters, Perpetuus Research \& Development Limited, EU Pat., EP3129323B1, 2019.

30 M. S. D. C. Dela Vega and M. R. Vasquez Jr, Plasmafunctionalized exfoliated multilayered graphene as cement reinforcement, Composites, Part B, 2019, 160, 573-585.

31 A. Dickschat and A. Studer, Radical Addition of Arylboronic Acids to Various Olefins under Oxidative Conditions, Org. Lett., 2010, 12, 3972-3974.

32 I. B. Seiple, S. Su, R. A. Rodriguez, R. Gianatassio, Y. Fujiwara, A. L. Sobel and P. S. Baran, Direct C-H Arylation of ElectronDeficient Heterocycles with Arylboronic Acids, J. Am. Chem. Soc., 2010, 132, 13194-13196.

33 Y. Fujiwara, V. Domingo, I. B. Seiple, R. Gianatassio, M. Del Bel and P. S. Baran, Practical $\mathrm{C}-\mathrm{H}$ Functionalization of Quinones with Boronic Acids, J. Am. Chem. Soc., 2011, 133, 3292-3295.

34 N. Uchiyama, E. Shirakawa, R. Nishikawa and T. Hayashi, Iron-catalyzed oxidative coupling of arylboronic acids with benzene derivatives through homolytic aromatic substitution, Chem. Commun., 2011, 47, 11671-11673.

35 D. Liu, C. Liu, H. Li and A. Lei, Direct Functionalization of Tetrahydrofuran and 1,4-Dioxane: Nickel-Catalyzed Oxidative $\mathrm{C}\left(\mathrm{sp}^{3}\right) \mathrm{H}$ Arylation, Angew. Chem., Int. Ed., 2013, 52, 4453-4456.

36 J. Y. Kim, S. Lee, T.-Y. Kim and H.-T. Kim, A Simple Diazonium Coupling Reaction Enhances Durability of Modified Graphitic 
Carbons Used as Catalyst Supports for Polymer Electrolyte Membrane Fuel Cell, Electrochim. Acta, 2014, 134, 418-425.

37 V. K. Kaushik, XPS core level spectra and Auger parameters for some silver compounds, J. Electron Spectrosc. Relat. Phenom., 1991, 56, 273-277.

38 J. C. Fuggle, E. Källne, L. M. Watson and D. J. Fabian, Electronic structure of aluminum and aluminum-noblemetal alloys studied by soft-X-ray and X-ray photoelectron spectroscopies, Phys. Rev. B: Solid State, 1977, 16, 750-761.

39 G. Zhang, M. Wen, S. Wang, J. Chen and J. Wang, Insights into thermal reduction of the oxidized graphite from the electro-oxidation processing of nuclear graphite matrix, $R S C$ Adv., 2018, 8, 567-579.

40 Y. Devrim and A. Albostan, Graphene-Supported Platinum Catalyst-Based Membrane Electrode Assembly for PEM Fuel Cell, J. Electron. Mater., 2016, 45, 3900-3907.

41 P.-G. Ren, D.-X. Yan, X. Ji, T. Chen and Z.-M. Li, Temperature dependence of graphene oxide reduced by hydrazine hydrate, Nanotechnology, 2011, 22(5), 055705.

42 F. Tuinstra and J. L. Koenig, Raman Spectrum of Graphite, J. Chem. Phys., 1970, 53, 1126-1130.

43 M. A. Pimenta, G. Dresselhaus, M. S. Dresselhaus, L. G. Cançado, A. Jorio and R. Saito, Studying disorder in graphite-based systems by Raman spectroscopy, Phys. Chem. Chem. Phys., 2007, 9, 1276-1291.

44 A. C. Ferrari and J. Robertson, Interpretation of Raman spectra of disordered and amorphous carbon, Phys. Rev. B: Condens. Matter Mater. Phys., 2000, 61, 14095-14107.

45 A. C. Ferrari, J. C. Meyer, V. Scardaci, C. Casiraghi, M. Lazzeri, F. Mauri, S. Piscanec, D. Jiang, K. S. Novoselov, S. Roth and A. K. Geim, Raman Spectrum of Graphene and Graphene Layers, Phys. Rev. Lett., 2006, 97, 187401.

46 V. T. Nguyen, H. D. Le, V. C. Nguyen, T. T. T. Ngo, D. Q. Le, X. N. Nguyen and N. M. Phan, Synthesis of multi-layer graphene films on copper tape by atmospheric pressure chemical vapor deposition method, Adv. Nat. Sci.: Nanosci. Nanotechnol., 2013, 4, 035012.

47 R. P. Vidano, D. B. Fischbach, L. J. Willis and T. M. Loehr, Observation of Raman band shifting with excitation wavelength for carbons and graphites, Solid State Commun., 1981, 39, 341-344.
48 D. C. Elias, R. R. Nair, T. M. G. Mohiuddin, S. V. Morozov, P. Blake, M. P. Halsall, A. C. Ferrari, D. W. Boukhvalov, M. I. Katsnelson, A. K. Geim and K. S. Novoselov, Control of Graphene's Properties by Reversible Hydrogenation: Evidence for Graphane, Science, 2009, 323, 610-613.

49 M. S. Seehra, U. K. Geddam, D. Schwegler-Berry and A. B. Stefaniak, Detection and quantification of $2 \mathrm{H}$ and $3 \mathrm{R}$ phases in commercial graphene-based materials, Carbon, 2015, 95, 818-823.

50 A. Bianco, H.-W. Cheng, T. Enoki, Y. Gogotsi, R. H. Hurt and N. Koratkar, All in the graphene family - A recommended nomenclature for two-dimensional carbon materials, Carbon, 2013, 65, 1-6.

51 K. S. Subrahmanyam, S. R. C. Vivekchand, A. Govindaraj and C. N. R. Rao, A study of graphenes prepared by different methods: characterization, properties and solubilization, J. Mater. Chem., 2008, 18, 1517-1523.

52 H. E. Swanson, R. K. Fuyat and G. M. Ugrinic, Circular. Standard X-ray Diffraction Powder Patterns, 1955, vol. IV, 539, p. 44 .

53 T. Barth and G. Lunde, Die Gitterkonstanten der Platinmetalle, Silber und Gold, Z. Phys. Chem., 1926, 121, 78-102.

54 B. N. Mehrotra, T. Hahn, W. Eysel, H. Roepke and A. Illguth, Polymorphism of sodium sulfate, $\mathrm{Na}_{2} \mathrm{SO}_{4}$, Neues Jahrb. Mineral., Monatsh., 1978, 408-421.

55 E. P. Barrett, L. G. Joyner and P. P. Halenda, The Determination of Pore Volume and Area Distributions in Porous Substances. I. Computations from Nitrogen Isotherms, J. Am. Chem. Soc., 1951, 73, 373-380.

56 F. Rouquerol, J. Rouquerol, K. S. W. Sing, G. Mourin and P. Llewellyn, Adsorption by Powders and Porous Solids, Elsevier, Amsterdam, 2nd edn, 2012.

57 M. Thommes, K. Kaneko, A. V. Neimark, J. P. Olivier, F. Rodriguez-Reinoso, J. Rouquerol and K. S. W. Sing, Physisorption of gases, with special reference to the evaluation of surface area and pore size distribution (IUPAC Technical Report), Pure Appl. Chem., 2015, 87, 1051-1069.

58 K. S. W. Sing, Reporting physisorption data for gas/solid systems with special reference to the determination of surface area and porosity (Recommendations 1984), Pure Appl. Chem., 1985, 57, 603-619. 